\title{
Several Integrability Formulas of Some Functions, Orthogonal Polynomials and Norm Functions
}

\author{
Bo $\mathrm{Li}$ \\ Qingdao University of Science \\ and Technology \\ China \\ Bing Xie \\ Qingdao University of Science \\ and Technology \\ China
}

\author{
Yanping Zhuang \\ Qingdao University of Science \\ and Technology \\ China \\ Pan Wang \\ Qingdao University of Science \\ and Technology \\ China
}

Summary. In this article, we give several integrability formulas of some functions including the trigonometric function and the index function [3]. We also give the definitions of the orthogonal polynomial and norm function, and some of their important properties [19].

MML identifier: INTEGRA9, version: $7.11 .01 \quad 4.117 .1046$

The terminology and notation used here are introduced in the following articles: [10], [21], [17], [6], [20], [1], [9], [13], [2], [4], [18], [15], [5], [8], [11], [14], [12], [16], and [7].

For simplicity, we use the following convention: $r, p, x$ denote real numbers, $n$ denotes an element of $\mathbb{N}, A$ denotes a closed-interval subset of $\mathbb{R}, f, g$ denote partial functions from $\mathbb{R}$ to $\mathbb{R}$, and $Z$ denotes an open subset of $\mathbb{R}$.

We now state a number of propositions:

(1) $\quad-$ (the function $\exp ) \cdot((-1) \square+0)$ is differentiable on $\mathbb{R}$ and for every $x$ holds (-(the function exp) $\cdot((-1) \square+0))^{\prime}(x)=\exp (-x)$. 
(2) $\int_{A}(($ the function $\exp ) \cdot((-1) \square+0))(x) d x=-\exp (-\sup A)+\exp (-\inf A)$.

(3) $\frac{1}{2}(($ the function $\exp ) \cdot(2 \square+0))$ is differentiable on $\mathbb{R}$ and for every $x$ holds $\left(\frac{1}{2}((\text { the function } \exp ) \cdot(2 \square+0))\right)_{\uparrow \mathbb{R}}^{\prime}(x)=\exp (2 \cdot x)$.

(4) $\int_{A}(($ the function exp $) \cdot(2 \square+0))(x) d x=\frac{1}{2} \cdot \exp (2 \cdot \sup A)-\frac{1}{2} \cdot \exp (2 \cdot \inf A)$.

(5) Suppose $r \neq 0$. Then $\frac{1}{r}$ ((the function exp) $\left.\cdot(r \square+0)\right)$ is differentiable on $\mathbb{R}$ and for every $x$ holds $\left(\frac{1}{r}((\text { the function exp }) \cdot(r \square+0))\right)^{\prime}(x)=\exp (r \cdot x)$.

(6) If $r \neq 0$, then $\int_{A}(($ the function $\exp ) \cdot(r \square+0))(x) d x=\frac{1}{r} \cdot \exp (r \cdot \sup A)-$ $\frac{1}{r} \cdot \exp (r \cdot \inf A)$.

(7) $\int_{A}(($ the function $\sin ) \cdot(2 \square+0))(x) d x=\left(-\frac{1}{2}\right) \cdot \cos (2 \cdot \sup A)-\left(-\frac{1}{2}\right) \cdot \cos (2 \cdot$ $\inf A)$.

(8) Suppose $n \neq 0$. Then $\left(-\frac{1}{n}\right)(($ the function $\cos ) \cdot(n \square+0))$ is differentiable on $\mathbb{R}$ and for every $x$ holds $\left(\left(-\frac{1}{n}\right)((\text { the function } \cos ) \cdot(n \square+0))\right)_{\mid \mathbb{R}}^{\prime}(x)=$ $\sin (n \cdot x)$.

(9) If $n \neq 0$, then $\int_{A}(($ the function $\sin ) \cdot(n \square+0))(x) d x=\left(-\frac{1}{n}\right) \cdot \cos (n \cdot$ $\sup A)-\left(-\frac{1}{n}\right) \cdot \cos (n \cdot \inf A)$.

(10) $\frac{1}{2}$ ((the function $\left.\left.\sin \right) \cdot(2 \square+0)\right)$ is differentiable on $\mathbb{R}$ and for every $x$ holds $\left(\frac{1}{2}((\text { the function } \sin ) \cdot(2 \square+0))\right)_{\uparrow \mathbb{R}}^{\prime}(x)=\cos (2 \cdot x)$.

(11) $\int_{A}(($ the function cos $) \cdot(2 \square+0))(x) d x=\frac{1}{2} \cdot \sin (2 \cdot \sup A)-\frac{1}{2} \cdot \sin (2 \cdot \inf A)$.

(12) Suppose $n \neq 0$. Then $\frac{1}{n}$ ((the function $\left.\left.\sin \right) \cdot(n \square+0)\right)$ is differentiable on $\mathbb{R}$ and for every $x$ holds $\left(\frac{1}{n}((\text { the function } \sin ) \cdot(n \square+0))\right)_{\uparrow \mathbb{R}}^{\prime}(x)=\cos (n \cdot x)$.

(13) If $n \neq 0$, then $\int_{A}(($ the function $\cos ) \cdot(n \square+0))(x) d x=\frac{1}{n} \cdot \sin (n \cdot \sup A)-$ $\frac{1}{n} \cdot \sin (n \cdot \inf A)$.

(14) If $A \subseteq Z$, then $\int_{A}\left(\operatorname{id}_{Z}(\right.$ the function $\left.\sin )\right)(x) d x=((-\sup A) \cdot \cos \sup A+$ $\sin \sup A)-((-\inf A) \cdot \cos \inf A+\sin \inf A)$.

(15) If $A \subseteq Z$, then $\int_{A}\left(\operatorname{id}_{Z}(\right.$ the function $\left.\cos )\right)(x) d x=(\sup A \cdot \sin \sup A+$ $\cos \sup A)-(\inf A \cdot \sin \inf A+\cos \inf A)$. 
(16) $\operatorname{id}_{Z}$ (the function cos) is differentiable on $Z$ and for every $x$ such that $x \in Z$ holds $\left(\operatorname{id}_{Z}\right.$ (the function $\left.\left.\cos \right)\right)_{{ }^{\prime}}^{\prime}(x)=\cos x-x \cdot \sin x$.

(17)(i) -the function $\sin +\mathrm{id}_{Z}$ (the function cos) is differentiable on $Z$, and

(ii) for every $x$ such that $x \in Z$ holds (-the function $\sin +\mathrm{id}_{Z}$ (the function $\cos ))_{\Gamma Z}^{\prime}(x)=-x \cdot \sin x$.

(18) If $A \subseteq Z$, then $\int_{A}\left(\left(-\mathrm{id}_{Z}\right)(\right.$ the function $\left.\sin )\right)(x) d x=(-\sin \sup A+\sup A$. $\cos \sup A)-(-\sin \inf A+\inf A \cdot \operatorname{cosinf} A)$.

(19)(i) -the function $\cos -\mathrm{id}_{Z}$ (the function $\sin$ ) is differentiable on $Z$, and

(ii) for every $x$ such that $x \in Z$ holds (-the function $\cos -\mathrm{id}_{Z}$ (the function $\sin ))^{\prime}{ }_{Z}(x)=-x \cdot \cos x$.

(20) If $A \subseteq Z$, then $\int_{A}\left(\left(-\mathrm{id}_{Z}\right)\right.$ (the function $\left.\left.\cos \right)\right)(x) d x=-\cos \sup A-\sup A$. $\sin \sup A-(-\cos \inf A-\inf A \cdot \sin \inf A)$.

(21) If $A \subseteq Z$, then $\int_{A}\left((\right.$ the function $\sin )+\operatorname{id}_{Z}($ the function $\left.\cos )\right)(x) d x=$ $\sup A \cdot \sin \sup A-\inf A \cdot \sin \inf A$.

(22) If $A \subseteq Z$, then $\int_{A}\left(-\right.$ the function $\cos +\operatorname{id}_{Z}($ the function $\left.\sin )\right)(x) d x=$ $(-\sup A) \cdot \cos \sup A-(-\inf A) \cdot \cos \inf A$.

(23) $\int_{A}((1 \square+0)($ the function $\exp ))(x) d x=\exp (\sup A-1)-\exp (\inf A-1)$.

(24) $\frac{1}{n+1}\left(\square^{n+1}\right)$ is differentiable on $\mathbb{R}$ and for every $x$ holds $\left(\frac{1}{n+1}\left(\square^{n+1}\right)\right)^{\prime}(x)=$ $x^{n}$.

(25) $\int_{A}\left(\square^{n}\right)(x) d x=\frac{1}{n+1} \cdot(\sup A)^{n+1}-\frac{1}{n+1} \cdot(\inf A)^{n+1}$.

(26) For all partial functions $f, g$ from $\mathbb{R}$ to $\mathbb{R}$ and for every non empty subset $C$ of $\mathbb{R}$ holds $(f-g)\lceil C=f\lceil C-g\lceil C$.

(27) For all partial functions $f_{1}, f_{2}, g$ from $\mathbb{R}$ to $\mathbb{R}$ and for every non empty subset $C$ of $\mathbb{R}$ holds $\left(\left(f_{1}+f_{2}\right) \uparrow C\right)\left(g\lceil C)=\left(f_{1} g+f_{2} g\right) \uparrow C\right.$.

(28) For all partial functions $f_{1}, f_{2}, g$ from $\mathbb{R}$ to $\mathbb{R}$ and for every non empty subset $C$ of $\mathbb{R}$ holds $\left(\left(f_{1}-f_{2}\right) \uparrow C\right)\left(g\lceil C)=\left(f_{1} g-f_{2} g\right) \uparrow C\right.$.

(29) For all partial functions $f_{1}, f_{2}, g$ from $\mathbb{R}$ to $\mathbb{R}$ and for every non empty subset $C$ of $\mathbb{R}$ holds $\left(\left(f_{1} f_{2}\right)\lceil C)\left(g\lceil C)=\left(f_{1}\lceil C)\left(\left(f_{2} g\right)\lceil C)\right.\right.\right.\right.$.

Let $A$ be a closed-interval subset of $\mathbb{R}$ and let $f, g$ be partial functions from $\mathbb{R}$ to $\mathbb{R}$. The functor $\langle f, g\rangle_{A}$ yielding a real number is defined by:

(Def. 1) $\langle f, g\rangle_{A}=\int_{A}(f g)(x) d x$. 
The following propositions are true:

(30) For all partial functions $f, g$ from $\mathbb{R}$ to $\mathbb{R}$ and for every closed-interval subset $A$ of $\mathbb{R}$ holds $\langle f, g\rangle_{A}=\langle g, f\rangle_{A}$.

(31) Let $f_{1}, f_{2}, g$ be partial functions from $\mathbb{R}$ to $\mathbb{R}$ and $A$ be a closed-interval subset of $\mathbb{R}$. Suppose that

(i) $\left(f_{1} g\right) \uparrow A$ is total,

(ii) $\left(f_{2} g\right) \uparrow A$ is total,

(iii) $\left(f_{1} g\right) \uparrow A$ is bounded,

(iv) $f_{1} g$ is integrable on $A$,

(v) $\left(f_{2} g\right) \uparrow A$ is bounded, and

(vi) $f_{2} g$ is integrable on $A$.

Then $\left\langle f_{1}+f_{2}, g\right\rangle_{A}=\left\langle\left(f_{1}\right), g\right\rangle_{A}+\left\langle\left(f_{2}\right), g\right\rangle_{A}$.

(32) Let $f_{1}, f_{2}, g$ be partial functions from $\mathbb{R}$ to $\mathbb{R}$ and $A$ be a closed-interval subset of $\mathbb{R}$. Suppose that

(i) $\left(f_{1} g\right) \uparrow A$ is total,

(ii) $\left(f_{2} g\right) \uparrow A$ is total,

(iii) $\left(f_{1} g\right) \uparrow A$ is bounded,

(iv) $f_{1} g$ is integrable on $A$,

(v) $\left(f_{2} g\right) \uparrow A$ is bounded, and

(vi) $f_{2} g$ is integrable on $A$.

Then $\left\langle f_{1}-f_{2}, g\right\rangle_{A}=\left\langle\left(f_{1}\right), g\right\rangle_{A}-\left\langle\left(f_{2}\right), g\right\rangle_{A}$.

(33) Let $f, g$ be partial functions from $\mathbb{R}$ to $\mathbb{R}$ and $A$ be a closed-interval subset of $\mathbb{R}$. Suppose $(f g)\lceil A$ is bounded and $f g$ is integrable on $A$ and $A \subseteq \operatorname{dom}(f g)$. Then $\langle-f, g\rangle_{A}=-\langle f, g\rangle_{A}$.

(34) Let $f, g$ be partial functions from $\mathbb{R}$ to $\mathbb{R}$ and $A$ be a closed-interval subset of $\mathbb{R}$. Suppose $(f g)\lceil A$ is bounded and $f g$ is integrable on $A$ and $A \subseteq \operatorname{dom}(f g)$. Then $\langle r f, g\rangle_{A}=r \cdot\langle f, g\rangle_{A}$.

(35) Let $f, g$ be partial functions from $\mathbb{R}$ to $\mathbb{R}$ and $A$ be a closed-interval subset of $\mathbb{R}$. Suppose $(f g)\lceil A$ is bounded and $f g$ is integrable on $A$ and $A \subseteq \operatorname{dom}(f g)$. Then $\langle r f, p g\rangle_{A}=r \cdot p \cdot\langle f, g\rangle_{A}$.

(36) For all partial functions $f, g, h$ from $\mathbb{R}$ to $\mathbb{R}$ and for every closed-interval subset $A$ of $\mathbb{R}$ holds $\langle f g, h\rangle_{A}=\langle f, g h\rangle_{A}$.

(37) Let $f, g$ be partial functions from $\mathbb{R}$ to $\mathbb{R}$ and $A$ be a closed-interval subset of $\mathbb{R}$. Suppose that $(f f)\lceil A$ is total and $(f g)\lceil A$ is total and $(g g) \uparrow$ $A$ is total and $(f f) \uparrow A$ is bounded and $(f g) \uparrow A$ is bounded and $(g g) \uparrow A$ is bounded and $f f$ is integrable on $A$ and $f g$ is integrable on $A$ and $g g$ is integrable on $A$. Then $\langle f+g, f+g\rangle_{A}=\langle f, f\rangle_{A}+2 \cdot\langle f, g\rangle_{A}+\langle g, g\rangle_{A}$.

Let $A$ be a closed-interval subset of $\mathbb{R}$ and let $f, g$ be partial functions from $\mathbb{R}$ to $\mathbb{R}$. We say that $f$ is orthogonal with $g$ in $A$ if and only if:

(Def. 2) $\langle f, g\rangle_{A}=0$. 
The following propositions are true:

(38) Let $f, g$ be partial functions from $\mathbb{R}$ to $\mathbb{R}$ and $A$ be a closed-interval subset of $\mathbb{R}$. Suppose that $(f f) \mid A$ is total and $(f g) \mid A$ is total and $(g g)\lceil A$ is total and $(f f) \uparrow A$ is bounded and $(f g) \uparrow A$ is bounded and $(g g) \uparrow A$ is bounded and $f f$ is integrable on $A$ and $f g$ is integrable on $A$ and $g g$ is integrable on $A$ and $f$ is orthogonal with $g$ in $A$. Then $\langle f+g, f+g\rangle_{A}=\langle f, f\rangle_{A}+\langle g, g\rangle_{A}$.

(39) Let $f$ be a partial function from $\mathbb{R}$ to $\mathbb{R}$ and $A$ be a closed-interval subset of $\mathbb{R}$. Suppose $(f f) \uparrow A$ is total and $(f f) \uparrow A$ is bounded and $f f$ is integrable on $A$ and for every $x$ such that $x \in A$ holds $((f f) \uparrow A)(x) \geq 0$. Then $\langle f, f\rangle_{A} \geq 0$.

(40) The function sin is orthogonal with the function $\cos$ in $[0, \pi]$.

(41) The function $\sin$ is orthogonal with the function $\cos$ in $[0, \pi \cdot 2]$.

(42) The function sin is orthogonal with the function $\cos$ in $[2 \cdot n \cdot \pi,(2 \cdot n+1) \cdot \pi]$.

(43) The function $\sin$ is orthogonal with the function $\cos$ in $[x+2 \cdot n \cdot \pi, x+$ $(2 \cdot n+1) \cdot \pi]$.

(44) The function $\sin$ is orthogonal with the function $\cos$ in $[-\pi, \pi]$.

(45) The function sin is orthogonal with the function $\cos$ in $\left[-\frac{\pi}{2}, \frac{\pi}{2}\right]$.

(46) The function $\sin$ is orthogonal with the function $\cos$ in $[-2 \cdot \pi, 2 \cdot \pi]$.

(47) The function sin is orthogonal with the function $\cos$ in $[-2 \cdot n \cdot \pi, 2 \cdot n \cdot \pi]$.

(48) The function $\sin$ is orthogonal with the function $\cos$ in $[x-2 \cdot n \cdot \pi, x+$ $2 \cdot n \cdot \pi]$.

Let $A$ be a closed-interval subset of $\mathbb{R}$ and let $f$ be a partial function from $\mathbb{R}$ to $\mathbb{R}$. The functor $\|f\|_{A}$ yields a real number and is defined by:

(Def. 3) $\quad\|f\|_{A}=\sqrt{\langle f, f\rangle_{A}}$.

Next we state three propositions:

(49) Let $f$ be a partial function from $\mathbb{R}$ to $\mathbb{R}$ and $A$ be a closed-interval subset of $\mathbb{R}$. Suppose $(f f)\lceil A$ is total and $(f f)\lceil A$ is bounded and $f f$ is integrable on $A$ and for every $x$ such that $x \in A$ holds $((f f) \uparrow A)(x) \geq 0$. Then $0 \leq\|f\|_{A}$.

(50) For every partial function $f$ from $\mathbb{R}$ to $\mathbb{R}$ and for every closed-interval subset $A$ of $\mathbb{R}$ holds $\|1 f\|_{A}=\|f\|_{A}$.

(51) Let $f, g$ be partial functions from $\mathbb{R}$ to $\mathbb{R}$ and $A$ be a closed-interval subset of $\mathbb{R}$. Suppose that $(f f) \uparrow A$ is total and $(f g) \uparrow A$ is total and $(g g) \uparrow A$ is total and $(f f) \uparrow A$ is bounded and $(f g) \uparrow A$ is bounded and $(g g)\lceil A$ is bounded and $f f$ is integrable on $A$ and $f g$ is integrable on $A$ and $g g$ is integrable on $A$ and $f$ is orthogonal with $g$ in $A$ and for every $x$ such that $x \in A$ holds $((f f) \uparrow A)(x) \geq 0$ and for every $x$ such that $x \in A$ holds $\left((g g)\lceil A)(x) \geq 0\right.$. Then $\left(\|f+g\|_{A}\right)^{2}=\left(\|f\|_{A}\right)^{2}+\left(\|g\|_{A}\right)^{2}$. 
For simplicity, we follow the rules: $a, b, x$ are real numbers, $n$ is an element of $\mathbb{N}, A$ is a closed-interval subset of $\mathbb{R}, f, f_{1}, f_{2}$ are partial functions from $\mathbb{R}$ to $\mathbb{R}$, and $Z$ is an open subset of $\mathbb{R}$.

Next we state several propositions:

(52) If $-a \notin A$, then $\frac{1}{1 \square+a}\lceil A$ is continuous.

(53) Suppose that

(i) $A \subseteq Z$,

(ii) for every $x$ such that $x \in Z$ holds $f(x)=a+x$ and $f(x) \neq 0$,

(iii) $Z=\operatorname{dom} f$,

(iv) $\operatorname{dom} f=\operatorname{dom} f_{2}$,

(v) for every $x$ such that $x \in Z$ holds $f_{2}(x)=-\frac{1}{(a+x)^{2}}$, and

(vi) $f_{2} \uparrow A$ is continuous.

Then $\int_{A} f_{2}(x) d x=f(\sup A)^{-1}-f(\inf A)^{-1}$.

(54) Suppose that

(i) $A \subseteq Z$,

(ii) for every $x$ such that $x \in Z$ holds $f(x)=a+x$ and $f(x) \neq 0$,

(iii) $\operatorname{dom}\left((-1) \frac{1}{f}\right)=Z$,

(iv) $\operatorname{dom}\left((-1) \frac{1}{f}\right)=\operatorname{dom} f_{2}$,

(v) for every $x$ such that $x \in Z$ holds $f_{2}(x)=\frac{1}{(a+x)^{2}}$, and

(vi) $f_{2} \uparrow A$ is continuous.

Then $\int_{A} f_{2}(x) d x=-f(\sup A)^{-1}+f(\inf A)^{-1}$.

(55) Suppose that

(i) $A \subseteq Z$,

(ii) for every $x$ such that $x \in Z$ holds $f(x)=a-x$ and $f(x) \neq 0$,

(iii) $\operatorname{dom} f=Z$,

(iv) $\operatorname{dom} f=\operatorname{dom} f_{2}$,

(v) for every $x$ such that $x \in Z$ holds $f_{2}(x)=\frac{1}{(a-x)^{2}}$, and

(vi) $f_{2} \uparrow A$ is continuous.

Then $\int_{A} f_{2}(x) d x=f(\sup A)^{-1}-f(\inf A)^{-1}$.

(56) Suppose that

(i) $A \subseteq Z$,

(ii) for every $x$ such that $x \in Z$ holds $f(x)=a+x$ and $f(x)>0$,

(iii) $\operatorname{dom}(($ the function $\ln ) \cdot f)=Z$,

(iv) $\operatorname{dom}(($ the function $\ln ) \cdot f)=\operatorname{dom} f_{2}$,

(v) for every $x$ such that $x \in Z$ holds $f_{2}(x)=\frac{1}{a+x}$, and

(vi) $f_{2} \uparrow A$ is continuous. 
Then $\int_{A} f_{2}(x) d x=\ln (a+\sup A)-\ln (a+\inf A)$.

Next we state a number of propositions:

(57) Suppose that

(i) $A \subseteq Z$,

(ii) for every $x$ such that $x \in Z$ holds $f(x)=x-a$ and $f(x)>0$,

(iii) $\operatorname{dom}(($ the function $\ln ) \cdot f)=Z$,

(iv) $\operatorname{dom}(($ the function $\ln ) \cdot f)=\operatorname{dom} f_{2}$,

(v) for every $x$ such that $x \in Z$ holds $f_{2}(x)=\frac{1}{x-a}$, and

(vi) $f_{2} \uparrow A$ is continuous.

Then $\int_{A} f_{2}(x) d x=\ln f(\sup A)-\ln f(\inf A)$.

(58) Suppose that

(i) $A \subseteq Z$,

(ii) for every $x$ such that $x \in Z$ holds $f(x)=a-x$ and $f(x)>0$,

(iii) $\operatorname{dom}(-($ the function $\ln ) \cdot f)=Z$,

(iv) $\operatorname{dom}(-($ the function $\ln ) \cdot f)=\operatorname{dom} f_{2}$,

(v) for every $x$ such that $x \in Z$ holds $f_{2}(x)=\frac{1}{a-x}$, and

(vi) $f_{2} \uparrow A$ is continuous.

Then $\int_{A} f_{2}(x) d x=-\ln (a-\sup A)+\ln (a-\inf A)$.

(59) Suppose that $A \subseteq Z$ and $f=($ the function $\ln ) \cdot f_{1}$ and for every $x$ such that $x \in Z$ holds $f_{1}(x)=a+x$ and $f_{1}(x)>0$ and $\operatorname{dom}\left(\operatorname{id}_{Z}-a f\right)=Z=$ $\operatorname{dom} f_{2}$ and for every $x$ such that $x \in Z$ holds $f_{2}(x)=\frac{x}{a+x}$ and $f_{2}\lceil A$ is continuous. Then $\int_{A} f_{2}(x) d x=\sup A-a \cdot f(\sup A)-(\inf A-a \cdot f(\inf A))$.

(60) Suppose that $A \subseteq Z$ and $f=($ the function $\ln ) \cdot f_{1}$ and for every $x$ such that $x \in Z$ holds $f_{1}(x)=a+x$ and $f_{1}(x)>0$ and $\operatorname{dom}\left((2 \cdot a) f-\operatorname{id}_{Z}\right)=$ $Z=\operatorname{dom} f_{2}$ and for every $x$ such that $x \in Z$ holds $f_{2}(x)=\frac{a-x}{a+x}$ and $f_{2}\lceil A$ is continuous. Then $\int_{A} f_{2}(x) d x=2 \cdot a \cdot f(\sup A)-\sup A-(2 \cdot a \cdot f(\inf A)-\inf A)$.

(61) Suppose that $A \subseteq Z$ and $f=($ the function $\ln ) \cdot f_{1}$ and for every $x$ such that $x \in Z$ holds $f_{1}(x)=x+a$ and $f_{1}(x)>0$ and $\operatorname{dom}\left(\operatorname{id}_{Z}-(2 \cdot a) f\right)=$ $Z=\operatorname{dom} f_{2}$ and for every $x$ such that $x \in Z$ holds $f_{2}(x)=\frac{x-a}{x+a}$ and $f_{2} \uparrow A$ is continuous. Then $\int_{A} f_{2}(x) d x=\sup A-2 \cdot a \cdot f(\sup A)-(\inf A-2 \cdot a \cdot f(\inf A))$.

(62) Suppose that $A \subseteq Z$ and $f=($ the function $\ln ) \cdot f_{1}$ and for every $x$ such that $x \in Z$ holds $f_{1}(x)=x-a$ and $f_{1}(x)>0$ and $\operatorname{dom}\left(\operatorname{id}_{Z}+(2 \cdot a) f\right)=$ $Z=\operatorname{dom} f_{2}$ and for every $x$ such that $x \in Z$ holds $f_{2}(x)=\frac{x+a}{x-a}$ and $f_{2} \uparrow A$ 
is continuous. Then $\int_{A} f_{2}(x) d x=(\sup A+2 \cdot a \cdot f(\sup A))-(\inf A+2 \cdot a \cdot$ $f(\inf A))$.

(63) Suppose that $A \subseteq Z$ and $f=($ the function $\ln ) \cdot f_{1}$ and for every $x$ such that $x \in Z$ holds $f_{1}(x)=x+b$ and $f_{1}(x)>0$ and $\operatorname{dom}\left(\operatorname{id}_{Z}+(a-b) f\right)=$ $Z=\operatorname{dom} f_{2}$ and for every $x$ such that $x \in Z$ holds $f_{2}(x)=\frac{x+a}{x+b}$ and $f_{2}\lceil A$ is continuous. Then $\int_{A} f_{2}(x) d x=(\sup A+(a-b) \cdot f(\sup A))-(\inf A+$ $(a-b) \cdot f(\inf A))$.

(64) Suppose that $A \subseteq Z$ and $f=($ the function $\ln ) \cdot f_{1}$ and for every $x$ such that $x \in Z$ holds $f_{1}(x)=x-b$ and $f_{1}(x)>0$ and $\operatorname{dom}\left(\operatorname{id}_{Z}+(a+b) f\right)=$ $Z=\operatorname{dom} f_{2}$ and for every $x$ such that $x \in Z$ holds $f_{2}(x)=\frac{x+a}{x-b}$ and $f_{2}\lceil A$ is continuous. Then $\int_{A} f_{2}(x) d x=(\sup A+(a+b) \cdot f(\sup A))-(\inf A+$ $(a+b) \cdot f(\inf A))$.

(65) Suppose that $A \subseteq Z$ and $f=($ the function $\ln ) \cdot f_{1}$ and for every $x$ such that $x \in Z$ holds $f_{1}(x)=x+b$ and $f_{1}(x)>0$ and $\operatorname{dom}\left(\operatorname{id}_{Z}-(a+b) f\right)=$ $Z=\operatorname{dom} f_{2}$ and for every $x$ such that $x \in Z$ holds $f_{2}(x)=\frac{x-a}{x+b}$ and $f_{2}\lceil A$ is continuous. Then $\int_{A} f_{2}(x) d x=\sup A-(a+b) \cdot f(\sup A)-(\inf A-(a+$ b) $\cdot f(\inf A))$.

(66) Suppose that $A \subseteq Z$ and $f=($ the function $\ln ) \cdot f_{1}$ and for every $x$ such that $x \in Z$ holds $f_{1}(x)=x-b$ and $f_{1}(x)>0$ and $\operatorname{dom}\left(\operatorname{id}_{Z}+(b-a) f\right)=$ $Z=\operatorname{dom} f_{2}$ and for every $x$ such that $x \in Z$ holds $f_{2}(x)=\frac{x-a}{x-b}$ and $f_{2}\lceil A$ is continuous. Then $\int_{A} f_{2}(x) d x=(\sup A+(b-a) \cdot f(\sup A))-(\inf A+$ $(b-a) \cdot f(\inf A))$.

(67) Suppose that

(i) $A \subseteq Z$,

(ii) for every $x$ such that $x \in Z$ holds $f(x)=x$ and $f(x)>0$,

(iii) $\operatorname{dom}(($ the function $\ln ) \cdot f)=Z$,

(iv) $\operatorname{dom}(($ the function $\ln ) \cdot f)=\operatorname{dom} f_{2}$,

(v) for every $x$ such that $x \in Z$ holds $f_{2}(x)=\frac{1}{x}$, and

(vi) $f_{2} \uparrow A$ is continuous.

Then $\int_{A} f_{2}(x) d x=\ln \sup A-\ln \inf A$.

(68) Suppose that

(i) $A \subseteq Z$,

(ii) for every $x$ such that $x \in Z$ holds $x>0$,

(iii) $\operatorname{dom}\left((\right.$ the function $\left.\ln ) \cdot\left(\square^{n}\right)\right)=Z$, 
(iv) $\operatorname{dom}\left((\right.$ the function $\left.\ln ) \cdot\left(\square^{n}\right)\right)=\operatorname{dom} f_{2}$,

(v) for every $x$ such that $x \in Z$ holds $f_{2}(x)=\frac{n}{x}$, and

(vi) $f_{2} \uparrow A$ is continuous.

Then $\int_{A} f_{2}(x) d x=\ln \left((\sup A)^{n}\right)-\ln \left((\inf A)^{n}\right)$.

(69) Suppose that

(i) $A \subseteq Z$,

(ii) for every $x$ such that $x \in Z$ holds $f(x)=x$,

(iii) $\operatorname{dom}\left((\right.$ the function $\left.\ln ) \cdot \frac{1}{f}\right)=Z$,

(iv) $\operatorname{dom}($ (the function $\left.\ln ) \cdot \frac{1}{f}\right)=\operatorname{dom} f_{2}$,

(v) for every $x$ such that $x \in Z$ holds $f_{2}(x)=-\frac{1}{x}$, and

(vi) $f_{2} \uparrow A$ is continuous.

Then $\int_{A} f_{2}(x) d x=-\ln \sup A+\ln \inf A$.

(70) Suppose that

(i) $A \subseteq Z$,

(ii) for every $x$ such that $x \in Z$ holds $f(x)=a+x$ and $f(x)>0$,

(iii) $\operatorname{dom}\left(\frac{2}{3} f^{\frac{3}{2}}\right)=Z$,

(iv) $\operatorname{dom}\left(\frac{2}{3} f^{\frac{3}{2}}\right)=\operatorname{dom} f_{2}$,

(v) for every $x$ such that $x \in Z$ holds $f_{2}(x)=(a+x)^{\frac{1}{2}}$, and

(vi) $f_{2} \uparrow A$ is continuous.

Then $\int_{A} f_{2}(x) d x=\frac{2}{3} \cdot(a+\sup A)^{\frac{3}{2}}-\frac{2}{3} \cdot(a+\inf A)^{\frac{3}{2}}$.

(71) Suppose that

(i) $A \subseteq Z$,

(ii) for every $x$ such that $x \in Z$ holds $f(x)=a-x$ and $f(x)>0$,

(iii) $\operatorname{dom}\left(\left(-\frac{2}{3}\right) f^{\frac{3}{2}}\right)=Z$,

(iv) $\operatorname{dom}\left(\left(-\frac{2}{3}\right) f^{\frac{3}{2}}\right)=\operatorname{dom} f_{2}$,

(v) for every $x$ such that $x \in Z$ holds $f_{2}(x)=(a-x)^{\frac{1}{2}}$, and

(vi) $f_{2} \uparrow A$ is continuous.

Then $\int_{A} f_{2}(x) d x=-\frac{2}{3} \cdot(a-\sup A)^{\frac{3}{2}}+\frac{2}{3} \cdot(a-\inf A)^{\frac{3}{2}}$.

(72) Suppose that

(i) $A \subseteq Z$,

(ii) for every $x$ such that $x \in Z$ holds $f(x)=a+x$ and $f(x)>0$,

(iii) $\operatorname{dom}\left(2 f^{\frac{1}{2}}\right)=Z$,

(iv) $\operatorname{dom}\left(2 f^{\frac{1}{2}}\right)=\operatorname{dom} f_{2}$,

(v) for every $x$ such that $x \in Z$ holds $f_{2}(x)=(a+x)^{-\frac{1}{2}}$, and

(vi) $f_{2} \uparrow A$ is continuous. 
Then $\int_{A} f_{2}(x) d x=2 \cdot(a+\sup A)^{\frac{1}{2}}-2 \cdot(a+\inf A)^{\frac{1}{2}}$.

(73) Suppose that

(i) $A \subseteq Z$,

(ii) for every $x$ such that $x \in Z$ holds $f(x)=a-x$ and $f(x)>0$,

(iii) $\operatorname{dom}\left((-2) f^{\frac{1}{2}}\right)=Z$,

(iv) $\operatorname{dom}\left((-2) f^{\frac{1}{2}}\right)=\operatorname{dom} f_{2}$,

(v) for every $x$ such that $x \in Z$ holds $f_{2}(x)=(a-x)^{-\frac{1}{2}}$, and

(vi) $f_{2} \uparrow A$ is continuous.

Then $\int_{A} f_{2}(x) d x=-2 \cdot(a-\sup A)^{\frac{1}{2}}+2 \cdot(a-\inf A)^{\frac{1}{2}}$.

(74) Suppose that

(i) $A \subseteq Z$,

(ii) $\operatorname{dom}\left(\left(-\mathrm{id}_{Z}\right)\right.$ (the function $\left.\cos \right)+$ the function $\left.\sin \right)=Z$,

(iii) for every $x$ such that $x \in Z$ holds $f(x)=x \cdot \sin x$,

(iv) $Z=\operatorname{dom} f$, and

(v) $f \uparrow A$ is continuous.

Then $\int_{A} f(x) d x=(-\sup A \cdot \cos \sup A+\sin \sup A)-(-\inf A \cdot \cos \inf A+$ $\sin \inf A)$.

(75) Suppose $A \subseteq Z$ and dom (the function sec) $=Z$ and for every $x$ such that $x \in Z$ holds $f(x)=\frac{\sin x}{(\cos x)^{2}}$ and $Z=\operatorname{dom} f$ and $f\lceil A$ is continuous. Then $\int_{A} f(x) d x=\sec \sup A-\operatorname{secinf} A$.

(76) Suppose $Z \subseteq \operatorname{dom}(-$ the function cosec). Then - the function cosec is differentiable on $Z$ and for every $x$ such that $x \in Z$ holds (-the function $\operatorname{cosec})_{\uparrow Z}^{\prime}(x)=\frac{\cos x}{(\sin x)^{2}}$.

(77) Suppose $A \subseteq Z$ and $\operatorname{dom}$ (-the function $\operatorname{cosec})=Z$ and for every $x$ such that $x \in Z$ holds $f(x)=\frac{\cos x}{(\sin x)^{2}}$ and $Z=\operatorname{dom} f$ and $f\lceil A$ is continuous. Then $\int_{A} f(x) d x=-\operatorname{cosec} \sup A+\operatorname{cosec} \inf A$.

\section{REFERENCES}

[1] Czesław Byliński. Partial functions. Formalized Mathematics, 1(2):357-367, 1990.

[2] Czesław Byliński and Piotr Rudnicki. Bounding boxes for compact sets in $\mathcal{E}^{2}$. Formalized Mathematics, 6(3):427-440, 1997.

[3] Chuanzhang Chen. Mathematical Analysis. Higher Education Press, Beijing, 1978.

[4] Noboru Endou and Artur Korniłowicz. The definition of the Riemann definite integral and some related lemmas. Formalized Mathematics, 8(1):93-102, 1999.

[5] Noboru Endou, Katsumi Wasaki, and Yasunari Shidama. Definition of integrability for partial functions from $\mathbb{R}$ to $\mathbb{R}$ and integrability for continuous functions. Formalized Mathematics, 9(2):281-284, 2001. 
[6] Krzysztof Hryniewiecki. Basic properties of real numbers. Formalized Mathematics, 1(1):35-40, 1990.

[7] Jarosław Kotowicz. Convergent sequences and the limit of sequences. Formalized Mathematics, 1(2):273-275, 1990.

[8] Jarosław Kotowicz. Partial functions from a domain to a domain. Formalized Mathematics, 1(4):697-702, 1990.

[9] Jarosław Kotowicz. Partial functions from a domain to the set of real numbers. Formalized Mathematics, 1(4):703-709, 1990.

[10] Jarosław Kotowicz. Real sequences and basic operations on them. Formalized Mathematics, 1(2):269-272, 1990.

[11] Konrad Raczkowski. Integer and rational exponents. Formalized Mathematics, 2(1):125130, 1991.

[12] Konrad Raczkowski and Paweł Sadowski. Real function differentiability. Formalized Mathematics, 1(4):797-801, 1990.

[13] Konrad Raczkowski and Paweł Sadowski. Topological properties of subsets in real numbers. Formalized Mathematics, 1(4):777-780, 1990.

[14] Yasunari Shidama. The Taylor expansions. Formalized Mathematics, 12(2):195-200, 2004.

[15] Andrzej Trybulec and Czesław Byliński. Some properties of real numbers. Formalized Mathematics, 1(3):445-449, 1990.

[16] Andrzej Trybulec and Yatsuka Nakamura. On the decomposition of a simple closed curve into two arcs. Formalized Mathematics, 10(3):163-167, 2002.

[17] Zinaida Trybulec. Properties of subsets. Formalized Mathematics, 1(1):67-71, 1990.

[18] Peng Wang and Bo Li. Several differentiation formulas of special functions. Part V. Formalized Mathematics, 15(3):73-79, 2007, doi:10.2478/v10037-007-0009-4.

[19] Renhong Wang. Numerical approximation. Higher Education Press, Beijing, 1999.

[20] Edmund Woronowicz. Relations defined on sets. Formalized Mathematics, 1(1):181-186, 1990.

[21] Yuguang Yang and Yasunari Shidama. Trigonometric functions and existence of circle ratio. Formalized Mathematics, 7(2):255-263, 1998.

Received October 14, 2008 